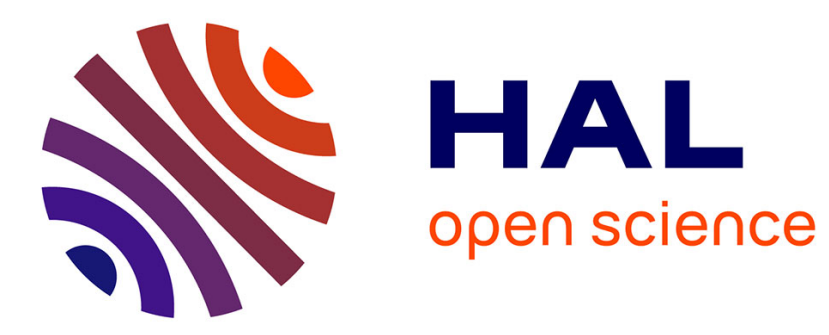

\title{
Relaxation of CDW polarization of various initial states
}

\author{
A. Beleznay, R. Gaal, G. Mihaly
}

\section{To cite this version:}

A. Beleznay, R. Gaal, G. Mihaly. Relaxation of CDW polarization of various initial states. Journal de Physique IV Proceedings, 1993, 03 (C2), pp.C2-357-C2-360. 10.1051/jp4:1993271 . jpa-00251354

\section{HAL Id: jpa-00251354 https://hal.science/jpa-00251354}

Submitted on 1 Jan 1993

HAL is a multi-disciplinary open access archive for the deposit and dissemination of scientific research documents, whether they are published or not. The documents may come from teaching and research institutions in France or abroad, or from public or private research centers.
L'archive ouverte pluridisciplinaire $\mathbf{H A L}$, est destinée au dépôt et à la diffusion de documents scientifiques de niveau recherche, publiés ou non, émanant des établissements d'enseignement et de recherche français ou étrangers, des laboratoires publics ou privés. 


\title{
Relaxation of CDW polarization of various initial states
}

\author{
A. BELEZNAY, R. GAAL and G. MIHALY \\ Research Institute for Solid State Physics, P.O. Box 49, 1525 Budapest, Hungary
}

\begin{abstract}
We have investigated the decay of the polarization induced by the application of electric field pulses of various lengths. Most experiments were carried out in $\mathrm{K}_{0.3} \mathrm{MoO}_{3}$ around $60 \mathrm{~K}$. In this case the characteristic time of the stretched exponential relaxation between two equilibrium states is a few ms. The pulse length was varied between $5 \mu \mathrm{s}<\Delta \mathrm{t}<10 \mathrm{~ms}$, and the resulting weak relaxation was detected over 7 decades in time. Our results resemble to the "aging effect" found in the low temperature thermal relaxation experiments, but we argue that both phenomena can be well described in the framework of a simple linear response theory.
\end{abstract}

The pinning of density waves to random lattice impurities allows the formation of metastable configurations separated by low energy barriers. As a consequence, various perturbations are followed by slow rearrangement of the condensate. For examples, electric properties revealed that thermal quench leads to long time decay of resistivity (1), or application of electric field step gives rise to weak relaxation in the polarization (2). Detailed low temperature specific heat studies also indicate a behaviour similar to that of glassy systems, furthermore the energy relaxation follow-ing heat pulses of different lengths resembles to the waiting time effects of spin glasses $(3,4)$.

In the present study we investigate the relaxation of charge density wave polarization induced by electric field pulses of various lengths, $\Delta t$. We found that the decay of the polarization spreads over several decades in time, the speed of the relaxation depends on the duration of field application and the behaviour exhibits strong temperature dependencies. We show that in spite of the apparent complexity of these observations the phenomenon can be well described in the framework of a simple linear response theory. Moreover, the relaxation of the polarization measured after electric field pulses of any finite length can simply be predicted from the asymptotic response for a step function.

The experiments were carried out in $\mathrm{K}_{0.3} \mathrm{MO}_{3}$ in the temperature range of $50-70 \mathrm{~K}$. Fig. 1a shows the decay of the normalized polarization, $P(t) / P_{0}$, as measured after electric field perturbations of various lengths. For short pulses the decay of the induced polarization is fast, while for longer ones the response saturates and approaches the stretched exponential response,

$$
\mathrm{P}(\mathrm{t}) / \mathrm{P}_{\mathrm{o}}=\exp -\left(\frac{\mathrm{t}}{\tau_{\mathrm{o}}}\right)^{\beta}
$$



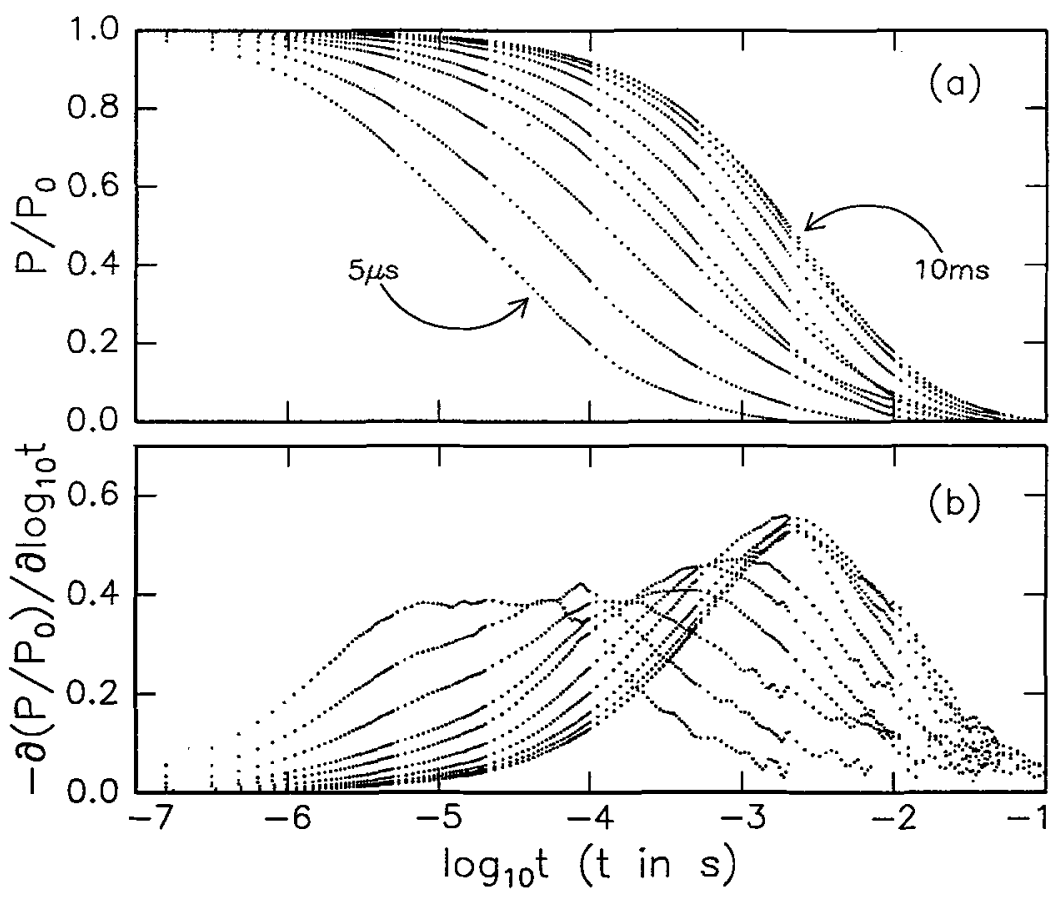

Fig. 1. Decay of the polarization induced by electric field pulses of various lengths $(\Delta \mathrm{t}=5,10,20,50,100,1000,5000$ and $10000 \mu \mathrm{s})$, and the logarithmic derivatives.
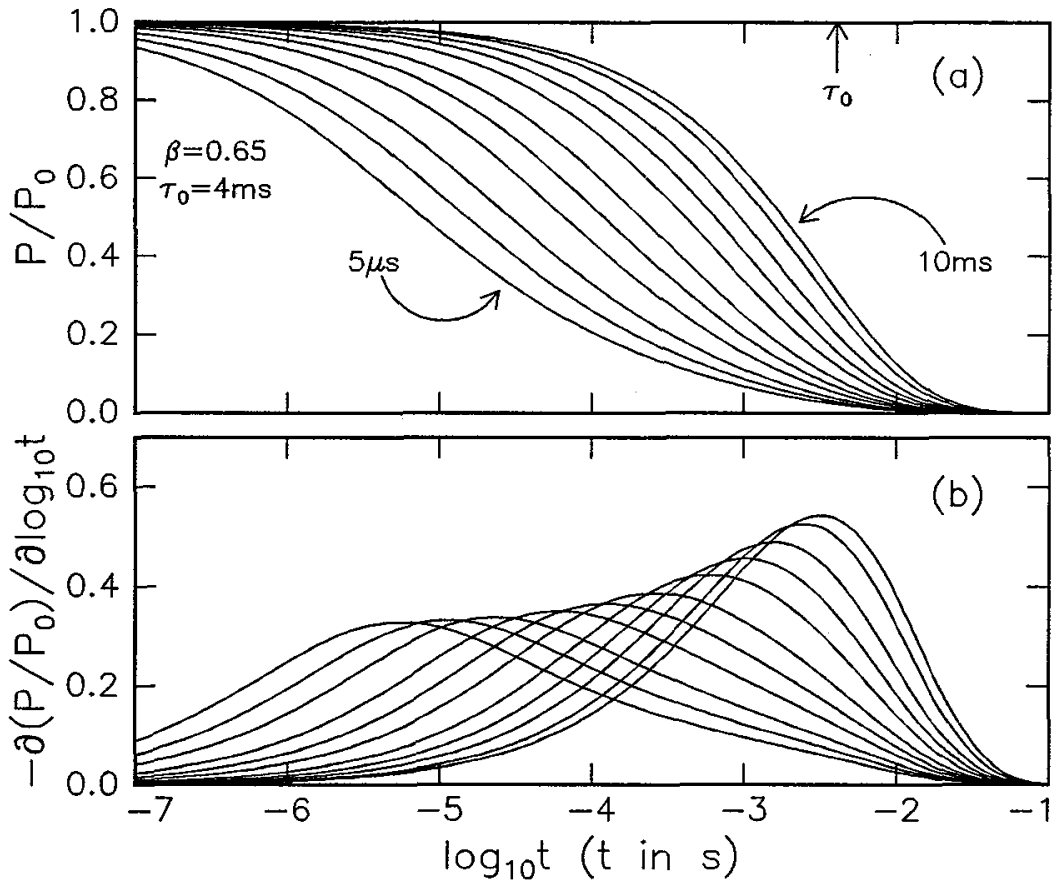

Fig. 2. Relaxation curves calculated by the application of linear response theory, with the assumption that the step response function is stretched exponential ( $\beta=0.65$ and $\tau=4 \mathrm{~ms}$ ). 
observed earlier for step function electric field perturbation (2). The logarithmic derivatives, shown on Fig. 1b, characterize the speed of the relaxation. The broad peak, often referred as average relaxation time, shifts to longer times as the pulse width is increased. As $\Delta t \Rightarrow \infty$, i.e. when the excitation tends to a step function, the logarithmic derivative approaches a well defined curve.

In the framework of the linear response theory this limiting curve can be related to the distribution function of the relaxation times, $g(\tau)$. For a simple Debye relaxation $g(\tau)=\delta\left(\tau-\tau_{\mathrm{o}}\right)$ with a single relaxation time, $\tau_{\mathrm{o}}$. If $\mathrm{g}(\tau)$ is broad then a step function excitation is followed by a nonexponentional relaxation,

$$
P(t)=P_{0} \int_{0}^{\infty} g(\ln \tau) \exp \{-t / \tau) d(\ln \tau) .
$$

It is important to realize that in this picture the distribution function is related solely to the excitation spectrum of the system and it is independent of the applied perturbation. With appropriate analysis $g(\tau)$ can be derived from the response to any form of perturbation, nevertheless the two simplest excitations are the step function and the harmonic excitation. In $\mathrm{K}_{0.3} \mathrm{MoO}_{3}$ both kind of experiments have been performed $(2,5)$ and the results are consistent with the linear response theory (6).

In the above picture the system is fully characterized by a single experiment and the relaxation following any perturbation can be deduced from the result of one measurement. Figure 2 shows a set of relaxation curves calculated from the assumption that application of an electric field step gives rise to a stretched exponential relaxation of $\mathrm{Eq}(1)$. The lengths of electric field perturbations used in this calculation are the same as the lengths of the pulses applied in the experiment shown on Fig.1. We emphasize that the parameters of $\mathrm{Eq}(1), \beta=0.65$ and $\tau_{\mathrm{o}}=4 \mathrm{~ms}$, are determined from the response for the longest electric field pulse and no fitting parameters have been used to calculate the 10 other response functions for pulse lengths from $5 \mu \mathrm{s}$ to $5 \mathrm{~ms}$.

The above simulation suggests that the difference in the relaxation curves can be explained by a single distribution function of the relaxation times and in order to interpret the change in the response when the pulse length is varied no further assumptions are necessary. As all the relaxation curves (and their logarithmic derivative) can be predicted from a single $\mathrm{g}(\tau)$, it is clear that the differences do not arise from variation of the distribution functions due to some aging effects. Our experiments thus demonstrate that in case of polarization relaxation there is no "waiting time effect", i.e. the application of electric field of various lengths does not change the barrier distribution of the system.

The logarithmic derivative of the relaxation curves, however, still can be used to characterize the speed of the relaxation. As the temperature is lowered the relaxation slows down [the parameter $\tau_{0}$ in Eq.(1) increases exponentially (2)]. For a constant pulse length the ratio $\Delta \mathrm{t} / \tau_{\mathrm{o}}$ decreases with decreasing temperature and this tendency corresponds to a shift towards shorter relative perturbation times in Fig. 2. Accordingly, if two pulses with different duration is applied the corresponding two peaks progressively separate with decreasing temperature. This is shown on Fig. 3, where the responses for $100 \mu \mathrm{s}$ and $10 \mathrm{~ms}$ pulses are shown at three different 


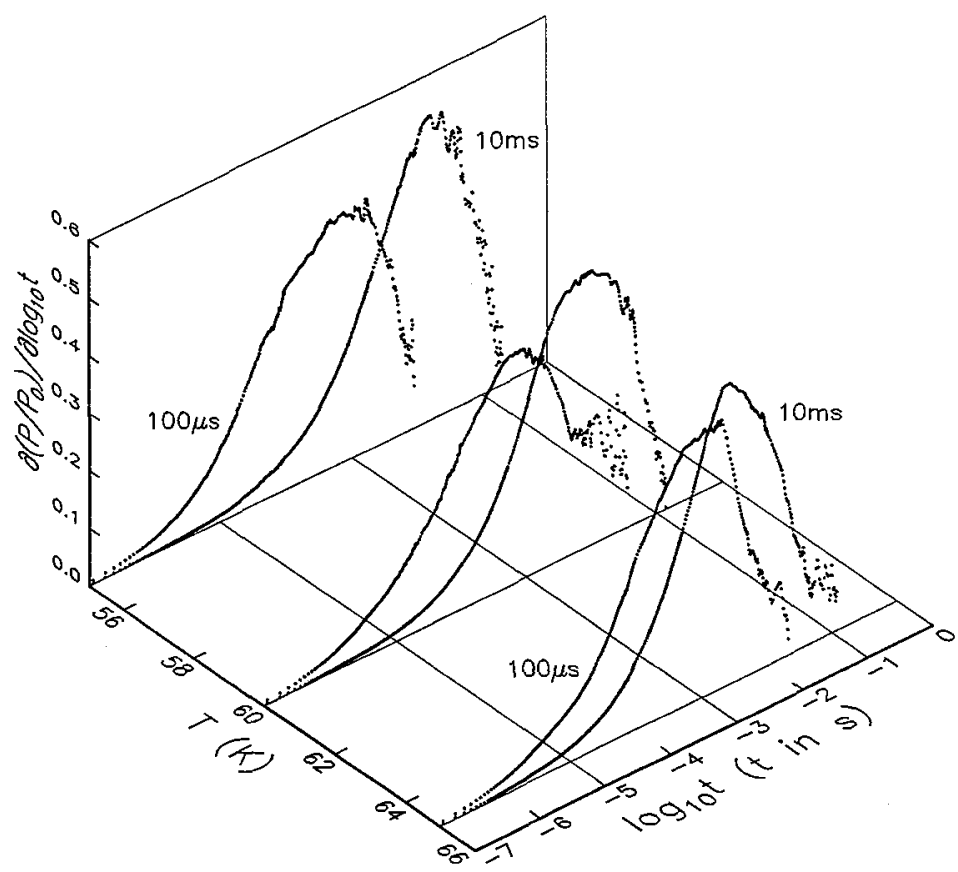

Fig. 3. The logarithmic derivatives of the polarization relaxation for $\Delta \mathrm{t}=100 \mu \mathrm{s}$ and $10 \mathrm{~ms}$ at three different temperatures.

temperatures. The curves strongly resembles to the time dependence of the relaxation rate observed in low temperature specific heat experiments (see Fig. 2 of Ref. 4).

In conclusion we have shown that nonexponentional relaxation phenomena of charge density wave polarization obey the linear response theory. There is no change in the structure of the metastable states during the application of the field and the logarithmic derivatives of the relaxation curves recorded after different excitations do not represent different distributions in the relaxation time. Recent analysis suggests (7) that the same interpretation applies for the low temperature specific heat experiments $(3,4)$, as well.

This work was supported by the Hungarian Scientific Research Foundation under Grant No. 2944 and 7277.

\section{References}

[1] Mihaly G.and Mihaly L., Phys. Rev. Lett. 52 (1984) 109.

[2] Kriza G. and Mihaly G., Phys. Rev. Lett. 56 (1986) 2529.

[3] Biljakovic K., Lasjaunias J. C., Monceau P. and Levy F., Phys. Rev. Lett. 62 (1989) 1512.

[4] Biljakovic K., Lasjaunias J. C., Monceau P. and Levy F., Phys. Rev. Lett. 67 (1991) 1902.

[5] Cava R. J., Fleming R. M., Littlewood P., Rietman E. A., Schneemeyer L. F., Dunn R. G. Phys. Rev. B30 (1984) 3228.

[6] Mihaly G. and Gruner G., Fizika 21 (1989) 71.

[7] Beleznay A. and Mihaly G., to be published. 